\title{
Exponential growth for wave equation with fractional boundary dissipation and boundary source term
}

Huiya Dai ${ }^{1,2^{*}}$ and Hongwei Zhang ${ }^{2}$

"Correspondence:

54214383@qq.com

'School of Mathematics and

Statistics, Xi'an Jiaotong University,

Xianning Road, Xi'an, P.R. China

${ }^{2}$ Department of Mathematics,

Henan University of Technology,

Lianhua Street, Zhengzhou,

P.R. China

\begin{abstract}
The wave equation with boundary source term and fractional boundary dissipation is considered. The exponential growth for sufficiently large initial data is proved. To this end some techniques based on Fourier transforms and some inequalities such as the Hardy-Littlewood-Soblev inequality are used.
\end{abstract}

Keywords: exponential growth; fractional boundary dissipation; wave equation

\section{Introduction}

In this paper we consider the unboundedness of the classical energy for the following problem:

$$
\begin{aligned}
& u_{t t}=\Delta u, \quad x \in \Omega, t>0, \\
& u=0, \quad x \in \Gamma_{0}, t>0, \\
& \frac{\partial u}{\partial v}+\partial_{t}^{\alpha} u=|u|^{m-1} u, \quad x \in \Gamma_{1}, t>0, \\
& u(x, 0)=u_{0}(x), \quad u_{t}(x, 0)=u_{1}(x), \quad x \in \Omega,
\end{aligned}
$$

where $\Omega$ is a bounded domain in $R^{n}$ with smooth boundary $\Gamma$ such that $\Gamma=\bar{\Gamma}_{0} \cap \bar{\Gamma}_{1}=\phi$, $m>1$ is a constant. The initial data $u_{0}(x)$ and $u_{1}(x)$ are given function, $\frac{\partial}{\partial \nu}$ denotes the outward normal derivative. The notation $\partial_{t}^{\alpha}$ stands for the Caputo fractional derivative of order $\alpha$ with respect to the time variable [1,2]. It is defined as follows:

$$
\partial_{t}^{\alpha} \omega(t)=\frac{1}{\Gamma(1-\alpha)} \int_{0}^{t}(t-s)^{-\alpha} \omega_{t}(s) d s, \quad 0<\alpha<1
$$

Let us mention here that the case $\alpha=0$ in (1)-(4) corresponds to a boundary damping and it has been extensively studied by many authors (see, for instance, [3-5] and references therein). It has been proved, in particular, that solutions exist globally in time when the initial data are in a stable set and the solution blows up in finite time when the initial data are in an unstable set.

The problem is motivated by some phenomena in viscoelasticity. Fractional differential systems have proved to be useful in control processing for the last two decades. Recently

\section{Springer}

(c) 2014 Dai and Zhang; licensee Springer. This is an Open Access article distributed under the terms of the Creative Commons Attribution License (http://creativecommons.org/licenses/by/4.0), which permits unrestricted use, distribution, and reproduction in any medium, provided the original work is properly credited. 
the linear wave equation with fractionally damped structures has received much attention (see $[2,6-10])$ and there has been a growing interest in investigating the solutions and properties of these evolution equation, where the source term vanish. But few contributions are concerned with the nonlinear or semi-linear wave equation with fractionally damped structure. This may be partly attributed to the fact that we do not benefit from a theoretical setting as convenient as the one provided by the semigroup theory [10]. Liang et al. [6] studied the boundary stabilization of a wave equation with fractional order boundary controller by symbolic algebra and numerical similarity. Mbodje [2] investigated the asymptotic behavior of solutions of the wave equation with a boundary viscoelastic damper of the fractional derivative type by semigroup theory. When the fractional order damped and sources term are the terms of the wave equation, Kirane and Tatar [11] and Tatar [12] proved that exponential growth and blow-up result for sufficient large initial data.

The fractional boundary dissipation can also be regarded as a viscosity term and boundary conditions of memory boundary terms. It is worth mentioning here that many authors have considered memory boundary terms (see [13-16] and references therein). However, in all these works the kernels appearing in their integral terms are all regular. In our case the kernel is not only singular but also non-integral. In this paper, we prove that the classical energy grows up exponentially when time goes to infinity by means of Fourier transforms and Hardy-Littlewood-Sobolev inequality. This technique has been used successfully by Kirane and Tatar [11] and Tatar [12] for the wave equation with fractional order damping. We also pointed out a similar problem with a fractional derivative term on part of its boundary which may blow up in finite time using the different method [17-20] and our main idea follows from [12].

The plan of the paper is as follows: in the next section we will prepare some materials needed to prove our result. Section 2 is devoted to the proof of our main result.

\section{Preliminaries}

Throughout this paper, we denote by $H^{m}(\Omega), L^{p}(\Omega)$ the usual Sobolev space, and

$$
(u, v)(t)=\int_{\Omega} u(x, t) v(x, t) d t, \quad\|u\|^{2}=(u, u)(t), \quad\|u\|_{p, \Gamma}^{p}=\int_{\Gamma}|u(x)|^{p} d \Gamma .
$$

Let us define

$$
E(t)=E(u(t))=\frac{1}{2}\left\|u_{t}\right\|^{2}+\frac{1}{2}\|\nabla u\|^{2}-\frac{1}{m+1}\|u\|_{m+1, \Gamma}^{m+1} .
$$

It is easily seen that

$$
\frac{d E(t)}{d t}=-\frac{1}{\Gamma(1-\alpha)} \int_{\Gamma} u_{t} \int_{0}^{t}(t-s)^{-\alpha} u_{t}(s) d s d \Gamma
$$

Observe that $\frac{d E(t)}{d t}$ is of an undefined sign and then the decreasing of the energy is not guaranteed. However, an integration of (6) with respect to time yields

$$
E(t)-E(0)=-\frac{1}{\Gamma(1-\alpha)} \int_{0}^{t} \int_{\Gamma} u_{t}(s) \int_{0}^{s}(s-z)^{-\alpha} u_{t}(z) d z d x d s \leq 0
$$


Hence $E(t)$ is uniformly bounded by $E(0)$, this is

$$
E(t) \leq E(0), \quad t \geq 0
$$

Lemma 2.1 (Hardy-Littlewood-Sobolev inequality, [21], p.354 in [22]) Let $u \in L^{p}(R), p>$ $1,0<\lambda<1$ and $\lambda>1-\frac{1}{p}$, then $\left(\frac{1}{|x|^{\lambda}}\right) * u \in L^{q}(R)$ with $\frac{1}{q}=\lambda+\frac{1}{p}-1$. Also the mapping from $u \in L^{p}(R)$ into $\left(\frac{1}{|x|^{\lambda}}\right) * u \in L^{q}(R)$ is continuous.

Lemma 2.2 ([23]) In the subspace

$$
V=\left\{v \in H^{1}(\Omega), v=0 \text { on } \Gamma_{0}\right\}
$$

of the Sobolev space $H^{1}(\Omega)$, there exists a constant $C_{0}>0$ such that

$$
\|v\|^{2} \leq C_{0}\|\nabla v\|^{2}, \quad v \in V
$$

Lemma $2.3([1,24])$ If we denote $\frac{t^{-\beta}}{\Gamma(1-\beta)}$ by $k_{\beta}(t)$, then we have

$$
k_{r+\eta-1}(t)=\left(k_{r} * k_{\eta}\right)(t), \quad 0<r, \eta<1 .
$$

\section{Exponential growth of the solution}

Theorem 3.1 Let $u(x, t)$ be a regular solution of (1)-(4). If the initial data $E(0)$ is large enough, $m>1$, then the solution $u(x, t)$ grows up exponentially in the $L^{m+1}(\Gamma)$-norm.

Proof Let us define

$$
G(t)=E(t)-\delta\left(u, u_{t}\right)
$$

where $0<\delta<1$ is a small constant to be determined later. Multiplying (1) by $u_{t}$ and then integrating over $\Omega$, we get

$$
\begin{aligned}
& \frac{d}{d t}\left(E(t)-\delta\left(u, u_{t}\right)\right)+\frac{1}{\Gamma(1-\alpha)} \int_{\Gamma} u_{t}(t) \int_{0}^{t}(t-s)^{-\alpha} u_{t}(s) d s d \Gamma \\
& \quad=\frac{\delta}{\Gamma(1-\alpha)} \int_{\Gamma} u \int_{0}^{t}(t-s)^{-\alpha} u_{t}(s) d s d \Gamma-\delta\left\|u_{t}\right\|^{2}-\delta\|u\|_{m+1, \Gamma}^{m+1}+\delta\|\nabla u\|^{2}
\end{aligned}
$$

For simplicity, we denote

$$
\begin{aligned}
& I_{1}=\frac{1}{\Gamma(1-\alpha)} \int_{0}^{T} u(s) \int_{0}^{s}(s-z)^{-\alpha} u_{t}(z) d s d z \\
& I_{2}=\frac{1}{\Gamma(1-\alpha)} \int_{0}^{T} u_{t}(s) \int_{0}^{s}(s-z)^{-\alpha} u_{t}(z) d s d z .
\end{aligned}
$$

Then, using the definition of $G(t)$ and integrating (8) over $[0, t]$ for $t$, we may write

$$
G(t)-G(0)+I_{2}=\delta I_{1}-\delta \int_{0}^{t}\left\|u_{t}\right\|^{2} d s-\delta \int_{0}^{t}\|u\|_{m+1, \Gamma}^{m+1} d s+\delta \int_{0}^{t}\|\nabla u\|^{2} d s
$$


Next, we estimate, for a fixed $t=T>0$,

$$
L_{T} \omega(\tau)= \begin{cases}\omega(\tau), & \tau \in[0, T] \\ 0, & \tau \in R-[0, T]\end{cases}
$$

and

$$
L \kappa_{\beta}(\tau):= \begin{cases}\kappa_{\beta}(\tau), & \tau>0 \\ 0, & \tau \leq 0\end{cases}
$$

where $\kappa_{\beta}(\tau)$ is as in Lemma 2.3. Then we can easily see that

$$
\begin{aligned}
I_{1} & =\int_{-\infty}^{+\infty} L_{T} u(s) \int_{-\infty}^{+\infty} L \kappa_{\alpha}(s-z) L_{T} u_{t}(z) d z d s \\
& =\int_{-\infty}^{+\infty} F\left(L_{T} u\right)(\sigma) \overline{F\left(L \kappa_{\alpha} * L_{T} u_{t}\right)}(\sigma) d \sigma,
\end{aligned}
$$

where we have used the Parseval theorem [21] and denoted by $F(f)$ the usual Fourier transform of $f$. Then, using Lemma 2.3, the Cauchy-Schwarz inequality, and the Young inequality, we obtain for $\rho_{1}>0$

$$
\begin{aligned}
I_{1} & \leq\left(\int_{-\infty}^{+\infty}\left|F\left(L \kappa_{\frac{\alpha+1}{2}}\right) F\left(L_{T} u\right)\right|^{2} d \sigma\right)^{\frac{1}{2}}\left(\int_{-\infty}^{+\infty}\left|F\left(L \kappa_{\frac{\alpha+1}{2}}\right) F\left(L_{T} u_{t}\right)\right|^{2} d \sigma\right)^{\frac{1}{2}} \\
& \leq \rho_{1} \int_{-\infty}^{+\infty}\left|F\left(L \kappa_{\frac{\alpha+1}{2}}\right) F\left(L_{T} u_{t}\right)\right|^{2} d \sigma+\frac{1}{4 \rho_{1}} \int_{-\infty}^{+\infty}\left|F\left(L \kappa_{\frac{\alpha+1}{2}}\right) F\left(L_{T} u\right)\right|^{2} d \sigma .
\end{aligned}
$$

For the last term in (11), we have

$$
\begin{aligned}
I & =\int_{-\infty}^{+\infty}\left|F\left(L \kappa_{\frac{\alpha+1}{2}}\right) F\left(L_{T} u\right)\right|^{2} d \sigma \leq \frac{1}{\cos \left(\frac{\alpha-1}{2} \pi\right)} \int_{-\infty}^{+\infty} L_{T} u(s)\left(L \kappa_{\alpha} * L_{T} u\right)(s) d s \\
& \leq \frac{1}{\cos \left(\frac{\alpha-1}{2} \pi\right)}\left(\int_{-\infty}^{+\infty}\left|L_{T} u\right|^{m+1} d s\right)^{\frac{1}{m+1}}\left(\int_{-\infty}^{+\infty}\left(L \kappa_{\alpha} * L_{T} u\right)^{\frac{m+1}{m}}(s) d s\right)^{\frac{m}{m+1}}
\end{aligned}
$$

here we have used the Hölder inequality and the following inequality [25, Theorem 16.5.1]:

$$
\int_{-\infty}^{+\infty}\left|F\left(\kappa_{\beta}\right) F(u)\right|^{2} d \sigma \leq \frac{1}{\cos ((\beta-1) \pi)} \int_{-\infty}^{+\infty} u(s)\left(k_{2 \beta-1} * u\right)(s) d s .
$$

Next we consider three cases.

(1) If $\frac{m}{m+1}<\alpha<1$, then the Lemma 2.1 with

$$
q=\frac{m}{m+1}, \quad \lambda=\alpha, \quad p=r=\frac{m+1}{2 m+1-\alpha(m+1)}>1
$$

implies that

$$
\left(\int_{-\infty}^{+\infty}\left(L \kappa_{(\alpha+1)} * L_{T} u\right)^{\frac{m+1}{m}}(s) d s\right)^{\frac{m}{m+1}} \leq \frac{C_{1}}{\Gamma(1-\alpha)}\left(\int_{-\infty}^{+\infty}\left|L_{T} u\right|^{r} d s\right)^{\frac{1}{r}},
$$

where $C_{1}=C_{1}(m, \alpha)$ depends only $m$ and $\alpha$. 
Since $r<2<m+1$, it is easily to see that

$$
\begin{aligned}
& \left(\int_{-\infty}^{+\infty}\left|L_{T} u\right|^{r} d s\right)^{\frac{1}{r}} \leq T^{\frac{m+1-r}{m+1}}\left(\int_{0}^{T}|u(s)|^{m+1} d s\right)^{\frac{1}{m+1}} \\
& \quad=T^{\frac{m+1-r}{m+1}}\left(\int_{-\infty}^{+\infty}\left|L_{T} u\right|^{m+1}(s) d s\right)^{\frac{1}{m+1}}
\end{aligned}
$$

Thus, from (12)-(14), we deduce that

$$
\begin{aligned}
I & \leq \frac{C_{1}}{\Gamma(1-\alpha) \cos \left(\frac{\alpha-1}{2} \pi\right)} T^{\frac{m+1-r}{m+1}}\left(\int_{-\infty}^{+\infty}\left|L_{T} u\right|^{m+1}(s) d s\right)^{\frac{1}{m+1}} \\
& \leq \frac{2}{m+1} C_{1}^{\frac{m+1}{2}}\left[\Gamma(1-\alpha) \cos \left(\frac{\alpha-1}{2} \pi\right)\right]^{-\frac{m+1}{2}} \int_{-\infty}^{+\infty}\left|L_{T} u\right|^{m+1} d s+\frac{m-1}{m+1} T^{\frac{m+1-r}{m+1}} .
\end{aligned}
$$

Using the estimate (15) in (11), we have

$$
\begin{aligned}
I_{1} \leq & \rho_{1} \int_{-\infty}^{+\infty}\left|F\left(L \kappa_{\frac{\alpha+1}{2}}(s-z)\right) F\left(L_{T} u_{t}\right)\right|^{2} d \sigma \\
& +\frac{M}{\rho_{1}} \int_{-\infty}^{+\infty}\left|L_{T} u\right|^{m+1} d s+\frac{m-1}{4 \rho_{1}(m+1)} T^{\frac{m+1-r}{m-1}} \\
= & \rho_{1} \int_{0}^{T}\left|\kappa_{\frac{\alpha+1}{2}} * u_{t}\right|^{2} d s+\frac{M}{\rho_{1}} \int_{0}^{T} u^{m+1} d s+\frac{m-1}{4 \rho_{1}(m+1)} T^{\frac{m+1-r}{m-1}}
\end{aligned}
$$

where $M=\frac{1}{2(m+1)}\left[\frac{\Gamma(1-\alpha)}{C_{1}} \cos \left(\frac{\alpha+1}{2} \pi\right)\right]^{-\frac{m+1}{2}}$. Analogously,

$$
\begin{aligned}
I_{2} & \leq \int_{-\infty}^{+\infty} L_{T} u_{t}(s) \int_{-\infty}^{+\infty} L \kappa_{\alpha}(s-z)\left(L_{T} u_{t}\right)(z) d z d s \\
& =\int_{-\infty}^{+\infty} F\left(L_{T} u_{t}\right)(\sigma) \overline{F\left(L \kappa_{\alpha} * L_{T} u_{t}\right)}(\sigma) d \sigma \\
& \leq \int_{-\infty}^{+\infty}\left|F\left(L \kappa_{\frac{\alpha+1}{2}}\right) F\left(L_{T} u_{t}\right)\right|^{2} d \sigma=\int_{0}^{T}\left|\kappa_{\frac{\alpha+1}{2}} * u_{t}\right|^{2} d \sigma .
\end{aligned}
$$

Hence, choosing $\rho_{1}=\frac{2 M(m+1)}{m-1}$ and taking into account (16), (17), and (9), we get

$$
\begin{aligned}
G(t)+\left[1-\delta \rho_{1}\right] \int_{\Gamma} \int_{0}^{T}\left|\kappa_{\frac{\alpha+1}{2}} * u_{t}\right|^{2} d s d \Gamma \\
\leq G(0)-\delta \int_{0}^{T}\left\|u_{t}\right\|^{2} d s+\delta \int_{0}^{T}\|\nabla u\|^{2} d s \\
+\frac{-(m+3)}{2(m+1)} \delta \int_{0}^{T}\|u\|_{m+1, \Gamma}^{m+1} d s+N T^{\sigma_{1}},
\end{aligned}
$$

where $N=\frac{\delta(m-1)^{2}|\Gamma|}{8 M(m+1)}$ and $\sigma_{1}=\frac{m+1-r}{m-1},|\Gamma|$ is the measure of $\Gamma$. 
Choosing $0<\delta<\frac{1}{\rho_{1}}$, we may reduce the inequality (18) to

$$
\begin{aligned}
G(T) \leq & G(0)+\frac{\delta(m+3)}{2} \int_{0}^{T} G(s) d s-\frac{m+8}{4} \delta \int_{0}^{T}\left\|u_{t}\right\|^{2}(s) d s \\
& +\frac{\delta^{2}(m+3)}{2} \int_{0}^{T}\left(u, u_{t}\right) d s+\frac{1-m}{4} \delta \int_{0}^{T}\|\nabla u\|^{2}(s) d s .
\end{aligned}
$$

By using the Cauchy-Schwarz inequality and the Poincaré inequality, we obtain

$$
\begin{aligned}
G(t) \leq & G(0)+\frac{\delta(m+3)}{2} \int_{0}^{T} G(s)+\frac{\delta}{8}\left[\frac{\delta(m+3)}{\rho}-\frac{2(m+7)}{4}\right] \int_{0}^{T}\left\|u_{t}\right\|^{2}(s) d s \\
& +\frac{\delta}{4}\left[2 \delta \rho C_{0}(m+3)-m+1\right] \int_{0}^{T}\|\nabla u\|^{2}(s) d s+N T^{\sigma_{1}},
\end{aligned}
$$

where $C_{0}$ is the Poincaré constant.

Clearly, it is possible to choose

$$
0<\delta<\min \left\{1, \frac{2(m+7) \rho}{m+3}, \frac{m-1}{2 \rho C_{0}(m+3)}\right\} .
$$

Thus the third and the fourth terms on the right-hand side of (20) are also negative. Then

$$
G(t) \leq G(0)+\frac{\delta(m+3)}{2} \int_{0}^{T} G(s) d s+N T^{\sigma_{1}}
$$

We define $\Phi(t)=-G(t)$. Clearly

$$
\Phi(T) \geq \Phi(0)+\frac{\delta(m+3)}{2} \int_{0}^{T} \Phi(s) d s-N T^{\sigma_{1}}
$$

Furthermore, we deduce from (22) that

$$
\begin{aligned}
\Phi(T) & \geq \Phi(0) e^{\frac{\delta(m+3) T}{2}}-\sigma_{1} N e^{\frac{\delta(m+3) T}{2}} \int_{0}^{T} s^{\sigma_{1}-1} e^{\frac{\delta(m+3) s}{2}} d s \\
& \geq\left\{\Phi(0)-\sigma_{1} N\left(\frac{\delta(m+3) T}{2}\right)^{-\sigma_{1}} \Gamma\left(\sigma_{1}\right)\right\} e^{\frac{\delta(m+3) T}{2}} .
\end{aligned}
$$

Since $E(0)$ is large enough, we can choose the initial data $u_{0}$ and $u_{1}$ such that

$$
\Phi(0)-\sigma_{1} N\left(\frac{\delta(m+3)}{2}\right)^{-\sigma_{1}} \Gamma\left(\sigma_{1}\right) \geq k>0,
$$

then we get

$$
\Phi(T) \geq k e^{\frac{\delta(m+3) T}{2}} .
$$

On the other hand, from the definition of $\Phi(t)$, the Cauchy-Schwarz inequality, and the Poincaré inequality, we see that

$$
\Phi(T) \leq \frac{1}{m+1}\|u\|_{m+1, \Gamma}^{m+1}-\frac{1-\delta}{2}\left\|u_{t}\right\|^{2}-\frac{1-\delta}{2}\|\nabla u\|^{2} .
$$


According to the choice of $\delta$ in (21), we get

$$
\Phi(T) \leq(1 /(m+1))\|u\|_{m+1, \Gamma}^{m+1}
$$

From the formulas (23) and (22) we conclude the exponential growth of the solution in the $L^{m+1}(\Gamma)$ norm.

(2) For the case $0<\alpha<\frac{m}{m+1}$, we note that

$$
\begin{aligned}
\left(L \kappa_{\alpha} * L_{T} u\right)(s) & =\frac{1}{\Gamma(1-\alpha)} \int_{0}^{s}(s-z)^{-\alpha} u(z) d z \\
& \leq C_{2}|s|^{1-\frac{\alpha(m+1)}{m}}\left(\int_{0}^{s}|u(z)|^{m+1} d z\right)^{\frac{m}{m+1}}
\end{aligned}
$$

where $C_{2}=\frac{1}{\Gamma(1-\alpha)}\left(1-\frac{\alpha(m+1)}{m}\right)^{-\frac{m}{m+1}}$ with $1-\frac{\alpha(m+1)}{m}>0$ (since $\left.0<\alpha<\frac{m}{m+1}\right)$. Taking this estimate into account in (12) and using the Young inequality, we find

$$
\begin{aligned}
I \leq & \frac{C_{2}}{\cos \left(\frac{\alpha-1}{2} \pi\right)}\left(\int_{0}^{T}|s|^{1-\frac{(\alpha+1)(m+1)}{2 m}} d s\right)^{\frac{m}{m+1}}\left(\int_{0}^{T}|u|^{m+1} d s\right)^{\frac{2}{m+1}} \\
\leq & \frac{m-1}{m+1}\left(2-\frac{(\alpha+1)(m+1)}{2 m}\right)^{\frac{-m}{m-1}} T^{1-\frac{(\alpha+1)(m+1)}{2(m-1)}} \\
& +\frac{2}{m+1}\left(\frac{C_{2}}{\cos \left(\frac{\alpha-1}{2} \pi\right)}\right)^{\frac{m+1}{2}} \int_{0}^{T}|u|^{m+1} d s .
\end{aligned}
$$

Using this estimate in (11) and proceeding as in part (1), we may conclude.

(3) If $\alpha=\frac{m}{m+1}$, we use the estimate

$$
I \leq \frac{1}{\cos \left(\frac{\alpha-1}{2} \pi\right)}\left(\int_{-\infty}^{+\infty}\left|L_{T} u\right|^{2} d s\right)^{\frac{1}{2}}\left(\int_{-\infty}^{+\infty}\left(L \kappa_{\frac{\alpha+1}{2}} * L_{T} u\right)^{2}(s) d s\right)^{\frac{1}{2}}
$$

In this case Lemma 2.1 is applicable with $q=2, \lambda=1-\frac{\alpha}{2}, p=r=\frac{2(m+1)}{2 m+1}$, we find

$$
I \leq \frac{C_{3}}{\Gamma(1-\alpha) \cos \left(\frac{\alpha-1}{2} \pi\right)}\left(\int_{0}^{T}|u|^{2} d s\right)^{\frac{1}{2}}\left(\int_{0}^{T}|u|^{r} d s\right)^{\frac{1}{r}} .
$$

Next, by the Hölder inequality and the Young inequality, we see that

$$
\begin{aligned}
I & \leq \frac{C_{3}}{\Gamma(1-\alpha) \cos \left(\frac{\alpha-1}{2} \pi\right)} T^{\frac{2 m-r}{m+1}}\left(\int_{0}^{T}|u|^{m+1} d s\right)^{\frac{2}{m+1}} \\
& \leq \frac{m-1}{m+1} T^{\frac{(2 m-r)}{(m+1)}}+\frac{1}{m+1}\left(\frac{C_{3}}{\Gamma(1-\alpha) \cos \left(\frac{\alpha-1}{2} \pi\right)}\right)^{\frac{m+1}{2}} \int_{0}^{T}|u|^{m+1} d s .
\end{aligned}
$$

This leads to an estimation of (12). Again the rest of the proof is similar to that in case (1). 


\section{Competing interests}

The authors declare that they have no competing interests.

\section{Authors' contributions}

The authors declare that the study was realized in collaboration with the same responsibility. All authors read and approved the final manuscript.

\section{Acknowledgements}

The first author wish to express sincere gratitude to his advisor Dr. Jigen Peng for his constructive and helpful suggestions and his encouragement in the pursuit of this work. This work is supported by National Natural Science Foundation of China (No. 11171311) and by the Natural Science Foundation of Henan Province (1323004100360).

\section{Received: 6 April 2014 Accepted: 24 May 2014 Published online: 20 September 2014}

\section{References}

1. Bajlekova, EG: Fractional Evolution Equations in Banach Spaces. Dissertation, Eindhoven University of Technology, Eindhoven (2001)

2. Mbodje, B: Wave energy decay under fractional derivative controls. IMA J. Math. Control Inf. 237, 237-257 (2006)

3. Vitillaro, E: Some new results on global nonexistence and blow up for evolution problem with positive initial energy. Rend. Ist. Mat. Univ. Trieste 31(2), 245-275 (2000)

4. Vitillaro, E: Global existence for the wave equation with boundary damping and source terms. J. Differ. Equ. 186, 259-298 (2002)

5. Zhang, HW, Hu, QY: Asymptotic behavior and nonexistence of wave equation with nonlinear boundary conditions. Commun. Pure Appl. Anal. 4(4), 861-869 (2005)

6. Liang, J, Chen, YQ, Vinagre, BM, Podlubny, I: Boundary stabilization of a fractional wave equation via a fractional order boundary controller.

http://www.researchgate.net/publication/228991225_Boundary_stabilization_of_a_fractional_wave_equation_ via_a_fractional_order_boundary_controller (2005)

7. Matignons, D, Audalnet, S, Montsney, G: Energy decay for wave equations with damping of fractional order. In: Proc of the Fourth International Conference on Mathematical and Numerical Aspects of Wave Propagation Phenomena, Golden, Colorado, June 1998, pp. 638-640 (1998)

8. Mbodje, B, Montsney, G: Boundary fractional derivative control of the wave equation. IEEE Trans. Autom. Control 40(2), 378-382 (1995)

9. Mbodje, B, Montsney, G, Audounet, J: Analysis of fractionally damped flexible systems via a diffusion equation. Int. J. Syst. Sci. 25(11), 1775-1791 (1994)

10. Mbodje, B, Montsney, G, Audounet, J, Benchimol, P: Optimal control for fractionally damped flexible systems. In: Proc. of the Third IEEE Conference on Control Applications, Strathclyde University, Glasgow, 24-26 August 1994, pp. 1329-1333 (1994)

11. Kirane, M, Tatar, N: Exponential growth for fractionally damped wave equation. Z. Anal. Anwend. 22(1), 167-177 (2003)

12. Tatar, N: A blow up result for a fractionally damped wave equation. Nonlinear Differ. Equ. Appl. 12, 215-226 (2005)

13. Kirane, $\mathrm{M}$, Tatar, N: A memory type boundary stabilization of a mildly damped wave equation. Electron. J. Qual. Theory Differ. Equ. 6, 1-7 (1999)

14. Kirane, $\mathrm{M}$, Tatar, N: Nonexistence results for a semilinear hyperbolic problem with boundary condition of memory type. Z. Anal. Anwend. 19(2), 1-16 (2000)

15. Aassila, M: Nonexistence of global solutions of a hyperbolic problem. Math. Comput. Model. 34, 761-769 (2001)

16. Aassila, M, Cavalcanti, MM, Soriano, JA: Asymptotic stability and energy decay rates for the solutions of the wave equation with memory in a star-shaped domain. SIAM J. Control Optim. 38, 1587-1602 (2000)

17. Labidi, S, Tatar, N: Unboundedness for the Euler-Bernoulli beam equation with a fractional boundary dissipation. Appl. Math. Comput. 161, 697-706 (2005)

18. Labidi, S, Tatar, N: Blow up for the Euler-Bernoulli beam problem with a fractional boundary dissipation. Dyn. Syst. Appl. 17(1), 109-119 (2008)

19. Labidi, S, Tatar, N: Blow-up of solutions for a nonlinear beam equation with fractional feedback. Nonlinear Anal. 74 , 1402-1409 (2011)

20. Lu, LQ, Li, SJ: Blow up of positive initial energy solutions for a wave equation with fractional boundary dissipation. Appl. Math. Lett. 24, 1729-1734 (2011)

21. Hormander, L: The Analysis of Linear Partial Differential Operators. I. Springer, Berlin (1983)

22. Stein, EM: Harmonic Analysis: Real-Variable Methods, Orthogonality, and Oscillatory Integrals. Princeton University Press, Princeton (1993)

23. Lions, JL, Magenes, E: Non-Homogeneous Boundary Value Problem and Applications. Springer, Berlin (1983)

24. Podlubny, l: Fractional Differential Equations. Mathematics in Science and Engineering, vol. 198. Academic Press, San Diego (1999)

25. Greenberg, G, Londen, SO, Staffans, O: Volterra Integral and Functional Equations. Cambridge University Press, Cambridge (1990)

doi:10.1186/s13661-014-0138-y

Cite this article as: Dai and Zhang: Exponential growth for wave equation with fractional boundary dissipation and boundary source term. Boundary Value Problems 2014 2014:138. 